\title{
Modeling hepatoblastoma development with human fetal liver organoids reveals YAP1 activation is sufficient for tumorigenesis
}

\section{Dear Editor,}

Hepatoblastoma $(\mathrm{HB})$ is a predominant hepatic neoplasm that develops in children from 0 to 4 years of age at the rate of 2.16 per $1,000,000$. It originates from abnormal differentiation of hepatocyte precursors (hepatoblasts) during embryogenesis (Sumazin et al., 2017). Approximately $20 \%$ of children with HB have metastasis in lung at diagnosis, which indicates poor prognosis (Angelico et al., 2019). While surgery in combination of chemotherapy and/or metastasectomy is the most popular therapy, relapse happens in a significant portion of HB patients (Zhang et al., 2021). Therefore, novel and less aggressive therapies targeting the pathogenesis of HB should be explored to prolong patients's disease-free survival as well as to improve their quality of life.

The in-depth understanding of the molecular mechanisms for HB progression is the key to develop target therapies. But the development of mechanistic studies for $\mathrm{HB}$ is rather slow due to the lack of authentic models. Currently, the only way to establish a HB model is to co-overexpress constitutively the constitutively active $\beta$-catenin (most frequently mutated gene) and its downstream target, Yes-associated protein 1 (Yap1), in adult mouse liver (Sylvester and Colnot, 2014). This model is quite different from the HB because: (1) its onset is in adult, and (2) there might be pathological differences between mouse and human HB.

Taking advantage of organoid technology, we employed a strategy to establish the human HB initiation model with fetal liver organoids. Based on the recently reported growth-factor-maintained fetal liver organoid culture system (Hu et al., 2018; Prior et al., 2019), we cultivated human fetal liver organoids using 8-12 weeks post-coitum (W) human liver cell clusters. The protocol was optimized by replacing the combination of growth factors with a small-molecule cocktail at day 7 for long-term expansion (Fig. 1A). The human fetal liver organoids retained the tissue-of-origin cell lineages and functions. We observed primary hepatocytes expressing hepatocyte nuclear factor 4 alpha (HNF4A), which can uptake low-density lipoprotein (LDL). We also identified primary bile canaliculi expressing multidrug resistance protein-
1 (MDR1), which can transport fluorescein diacetate (Fig. S1). Importantly, the organoids still maintained hepatoblast features including the robust expression of alphafetoprotein (AFP) and delta-like 1 homolog (DLK1) (Fig. 1B).

We then determined whether the active forms of $\beta$-catenin $\left(\beta\right.$-catenin ${ }^{\Delta \mathrm{ex} 3}$ ) (Sun et al., 2019) or YAP1 (YAP1 ${ }^{5 \mathrm{SA}}$ ) (Zhao et al., 2007) can induce HB oncogenesis in fetal liver organoids. Although both Wnt- $\beta$-catenin activation and HippoYAP activation (Fig. 1C) could promote the proliferation of human fetal liver organoids, only Hippo-YAP activation led to aggressive malignancy (Figs. 1D and S2).

We further dissected the downstream signaling of HippoYAP. It has been reported that YAP1 facilitates the nuclear location of $\beta$-catenin to activate Wnt- $\beta$-catenin signaling (Tao et al., 2014). In consistence, we observed that Wnt- $\beta$-catenin activation in YAP1 ${ }^{5 \mathrm{SA}}$-transfected human liver fetal organoids led to significant up-regulation of Wnt- $\beta$-catenin target genes (Gene Set Enrichment Analysis (GSEA) in Fig. S3A and $\mathrm{qPCR}$ in Fig. S3B). However, suppression of $\beta$-catenin transcriptional activity by IWP-2 treatment had no obvious effect on YAP1 ${ }^{5 S A}$-driven HB progression (Figs. $1 \mathrm{C}$ and S4), which demonstrated that Hippo-YAP activation is sufficient for human HB initiation, and that its effects are independent of Wnt- $\beta$-catenin signaling. These results suggest that the Hippo-YAP activation, induced by $W n t / \beta$-catenin activation in patients with $\beta$-catenin mutations, is the key event for HB initiation.

To validate that the YAP1-activated human fetal liver organoids obtained the key features of $\mathrm{HB}$, we subjected transformed organoids to transcriptome analysis and found 2,176 differentially expressed genes (DEGs) $(P<0.05$ and fold change $\geq 2$ ) (Fig. 1E). Particularly, Hippo-YAP activation induced the expression of several critical genes for $\mathrm{HB}$ oncogenesis, including DKK1, COL2A1, THFRSF19, NPNT, MATN3, CST1, PCP4, EDN3, C9orf152, and PEG10 (Fig. $1 \mathrm{E}$ and $\mathrm{QPCR}$ verification in Fig. 1F). GSEA revealed that YAP1-activated organoids significantly increased the expression of "HB signature genes" (Fig. 1G). These data indicated that Hippo-YAP activation transformed fetal liver organoids to HB organoids. 

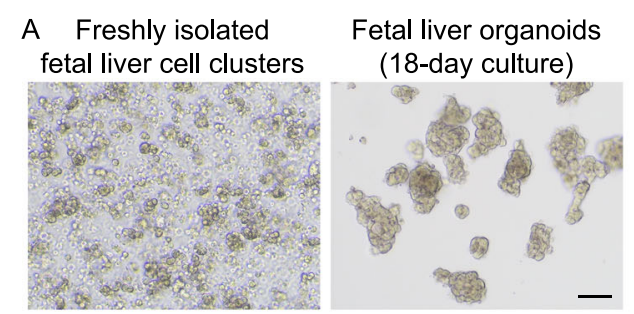

C

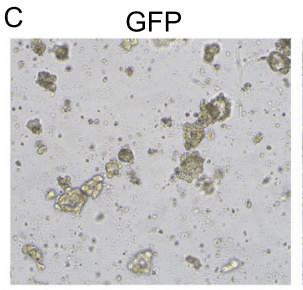

YAP15SA

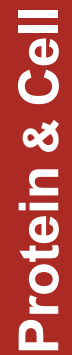

$\beta-$ Catenin $^{\Delta \mathrm{ex} 3}$

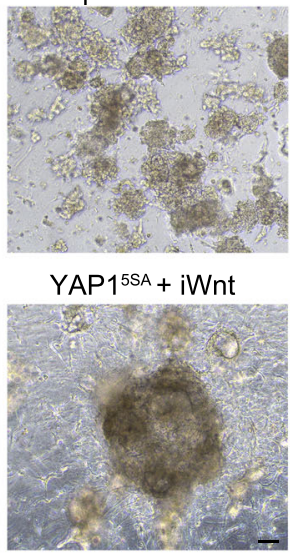

F
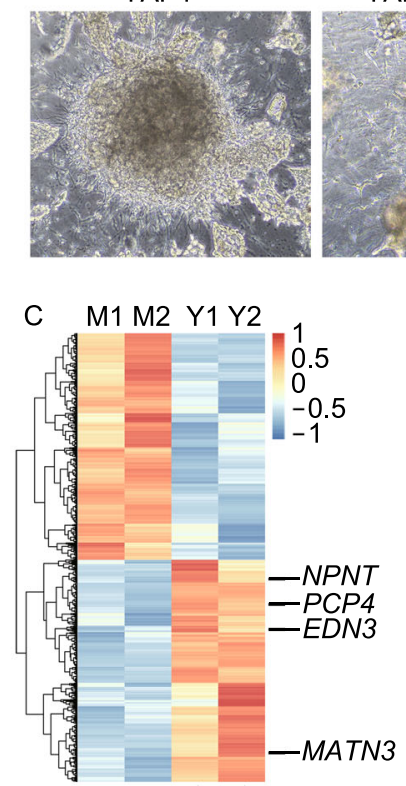

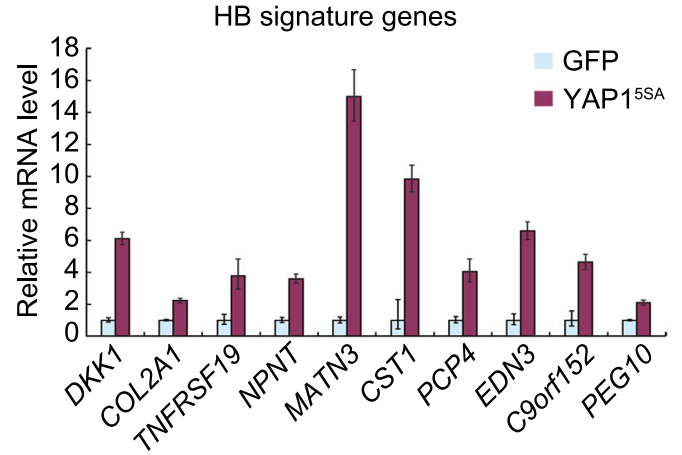

B Organoid cross-section staining AFP/DAPI

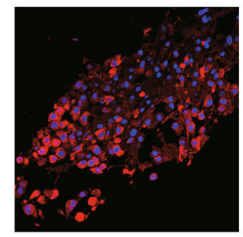
DLK1/DAPI

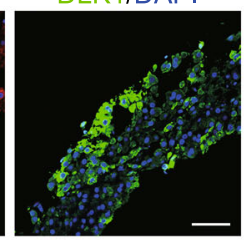

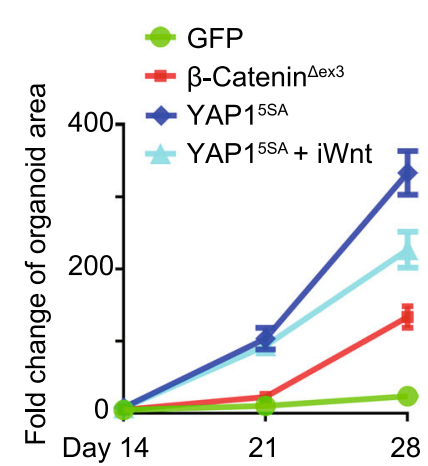

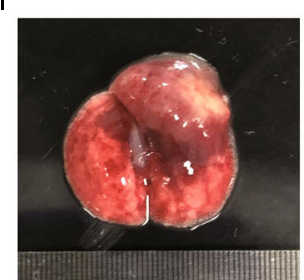

AFP/DAPI

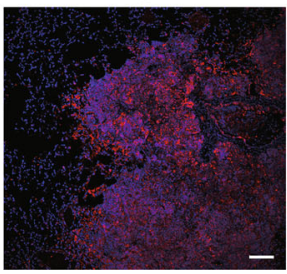

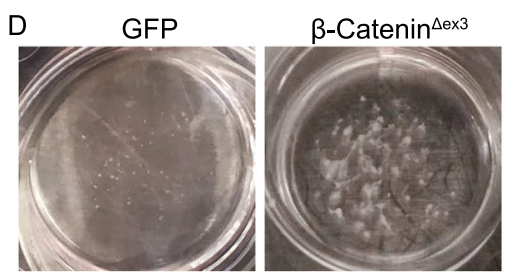

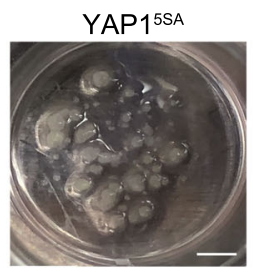

G

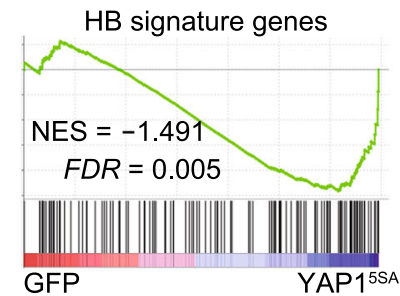

HB down-regulated genes

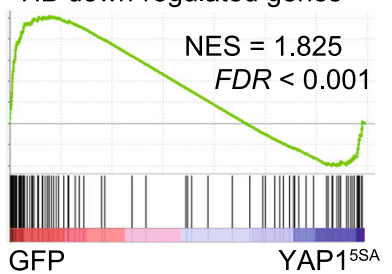

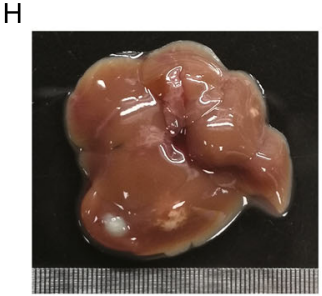

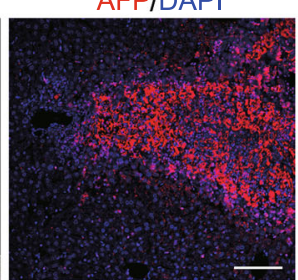

YAP1/DAPI
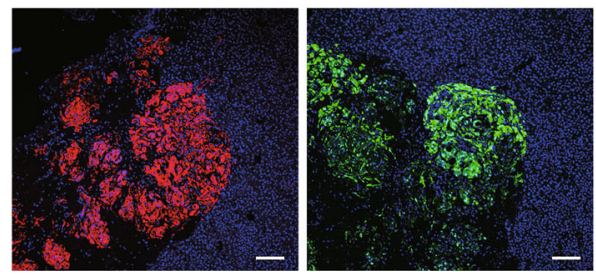

H\&E

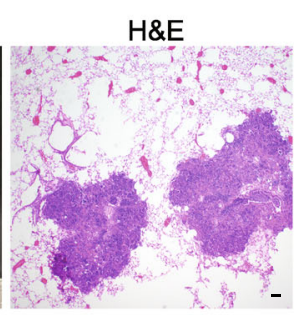

YAP1/DAPI

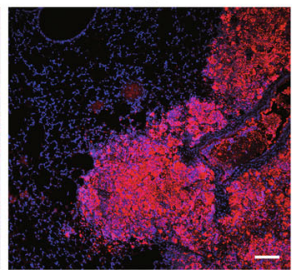


4 Figure 1. Modeling hepatoblastoma development with human fetal liver organoids reveals YAP1 activation is sufficient for tumorigenesis. (A) Brightfield images of freshly isolated human fetal liver cell clusters and 18-day cultured organoids. Scale bar, $200 \mu \mathrm{m}$. (B) Immunofluorescence staining for the hepatoblast markers AFP and DLK1. Scale bar, $50 \mu \mathrm{m}$. (C) Brightfield images of 21-day cultured fetal liver organoids transfected with lentiviral GFP, $\beta$-catenin ${ }^{\triangle \mathrm{ex} 3}$ or YAP $1^{5 \mathrm{SA}}$. Growth rate of organoids were quantified. iWnt: Wnt inhibition by $5 \mu \mathrm{mol} / \mathrm{L}$ IWP-2 treatment. Scale bar, $200 \mu \mathrm{m}$. (D) Organoids transfected with indicated genes were cultured for 60 days. Scale bar, $250 \mathrm{~mm}$. (E) mRNA expression heatmap of differentially expressed genes for Mock (M1, M2) and YAP1activated (Y1, Y2) organoids. (F) GFP and YAP1-transfected organoids were harvested to examine the expression of $\mathrm{HB}$ signature genes using qRT-PCR. $\mathrm{H} 3$ was used as an internal control. Data were presented as means $\pm \mathrm{SD}(n=3)$. (G) GSEA enrichment analysis of Mock versus YAP1-activated organoids for $\mathrm{HB} /$ normal top 200 genes (top) and $\mathrm{HB}$ /normal last 200 genes (bottom). ( $\mathrm{H}$ and I) YAP1-activated HB organoids were transplanted into the liver capsule of NSG mice. Tumors in liver $(\mathrm{H})$ and metastatic foci in lung $(\mathrm{I})$ were subjected to immunofluorescence staining for AFP, KRT19 and YAP1. Scale bar, 100 $\mu \mathrm{m}$.

In advance, we tested whether the Hippo-YAP activation induced malignancy by performing orthotopic liver transplantation of fetal liver organoids. In comparison to the control condition, YAP1-activated HB organoids gave rise to xenografts with high expression levels of HB histopathological markers AFP, YAP1, and keratin 19 (KRT19) in 16 out of 22 NOD-Prkdc ${ }^{\text {scid }} / 12 \mathrm{rg}^{\mathrm{em1}} / \mathrm{Smoc}$ (NSG) mice (Fig. 1H). Importantly, 5 of the 16 mice with liver HB exhibited spontaneous lung metastasis thus died (Fig. 1I and survival curve in Fig. S5), which well recapitulated the clinical characteristics of HB (O'Neill et al., 2017). The tumor tissues in lung retained the high expression levels of AFP, YAP1, and NuMA, which confirms that the metastatic foci originated from YAP1-activated human HB organoids (Figs. 1I and S6). Together, these results demonstrated that Hippo-YAP activation in fetal liver organoids well models the HB oncogenesis as well as the malignant progression.

Based on the modeling system, we tried to elucidate the detailed mechanism of how Hippo-YAP activation initiates HB. Several studies have suggested that Hippo-YAP activation induces metabolic reprogramming in hepatocytes. We therefore assessed the metabolic changes of the malignant organoids with LC-MS-based metabolomics. Interestingly, YAP1 activation changed one-carbon metabolism, as Sadenosylmethionine (SAM) was down-regulated while Sadenosyl-L-homocysteine (SAH) was up-regulated in YAP1activated organoids (Fig. 2A). This result indicated that methyltransferases activity alternation might disturb the balance between SAM and SAH in HB. Moreover, a panmethyltransferase inhibitor ADOX specifically inhibited the growth of YAP1 ${ }^{5 \mathrm{SA}}$-transfected organoids but not GFPtransfected organoids, suggesting the critical role of methyltransferase in Hippo-YAP-mediated transformation (Fig. 2B). We then scanned a small collection of methyltransferase inhibitors with four DNA methyltransferase inhibitors and 13 protein methyltransferase inhibitors on YAP1activated organoids. Notably, 4 out of the 6 inhibitors of histone methyltransferase (HMT) G9a (encoded by EHMT2), represented by BIX 01294, could specifically attenuate the growth of YAP1-activated organoids (Figs. 2C and S7).

EHMT2/G9a was up-regulated in both HB (Figs. 2D and S8) and HB organoids (Fig. 2E and 2F). Particularly, overexpression of G9a but not its enzyme-dead mutant drove $\mathrm{HB}$ oncogenesis in fetal liver organoids (Fig. 2G). In contrast, knock down of EHMT2 resulted in growth inhibition of $\mathrm{HB}$ organoids (Fig. $2 \mathrm{H}$ ), which could be rescued by shRNA-resistant EHMT2 overexpression (Fig. 2I). In addition, EHMT2 knockdown suppressed HB signature genes in YAP1-activated organoids (Fig. 2J).

Finally, we explored the potential therapeutic effects of methyltransferase inhibitors on HB. We subjected YAP1transformed $\mathrm{HB}$ organoids to the pan-methyltransferase inhibitor ADOX and found that methyltransferase inhibition significantly inhibited the growth of $\mathrm{HB}$ organoids at D40 (Fig. 2K). We also determined whether G9a inhibition could target $\mathrm{HB}$ in vivo by injecting G9a inhibitor BIX 01294 intraperitoneally. As shown in Fig. 2L, G9a inhibition dramatically decreased the size of xenografts one month following the orthotopic transplantation of $\mathrm{HB}$ organoids (Fig. 2L), which demonstrated the YAP1-G9a axis is a potential therapeutic target for $\mathrm{HB}$.

In summary, we established a human HB oncogenesis model by manipulating Hippo-YAP signaling in human fetal liver organoids. This model revealed that YAP1 activation is sufficient for human HB tumorigenesis, which overturns the traditional theory that both $\beta$-catenin and YAP1 activation are required (Driskill and Pan, 2021; Zhang et al., 2021). Mechanistic studies ex vivo and in vivo demonstrated that the histone methyltransferase G9a up-regulation is critical for $\mathrm{HB}$ oncogenesis. These results highlight the importance of metabolism-epigenetic regulation in $\mathrm{HB}$ and suggest that YAP1-G9a could be targeted to develop novel therapeutics.

\section{FOOTNOTES}

This work was supported by grants from the National Key Research and Development Program of China (2018YFA0109400), the National Natural Science Foundation of China (Grant Nos. 3202202 and 32071138), and the Open Research Fund of State Key Laboratory of Genetic Engineering of Fudan University (No. SKLGE2118).

The authors declare no conflict of interest.

All procedures followed were in accordance with the ethical standards of the Medical Ethical Council of Obstetrics and 

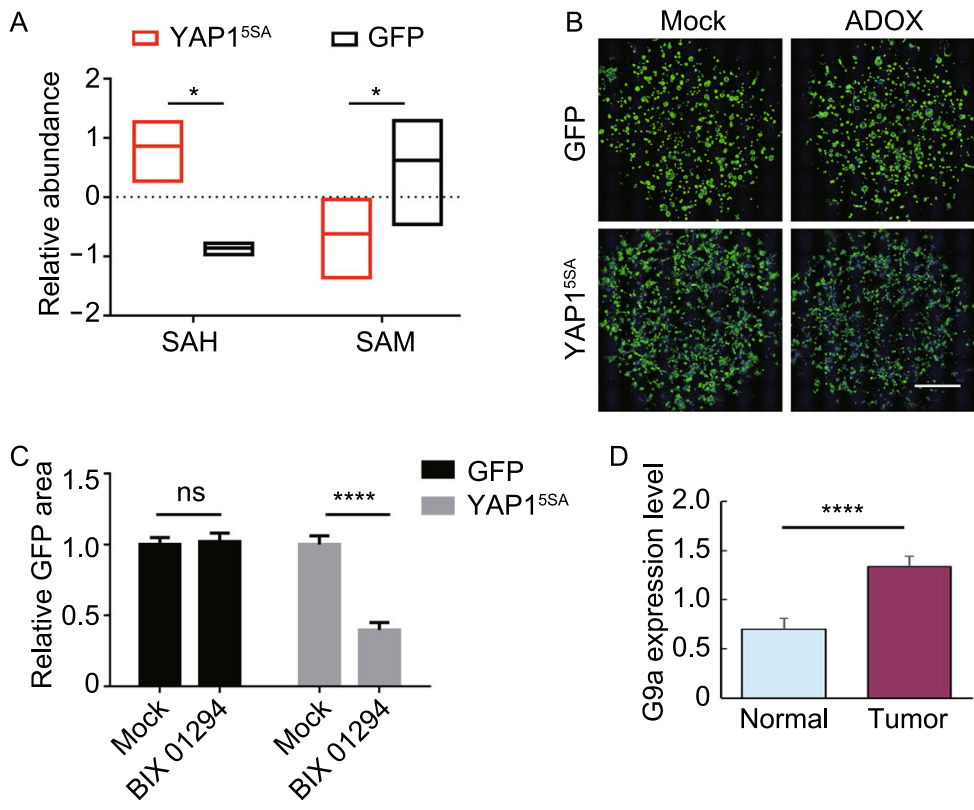
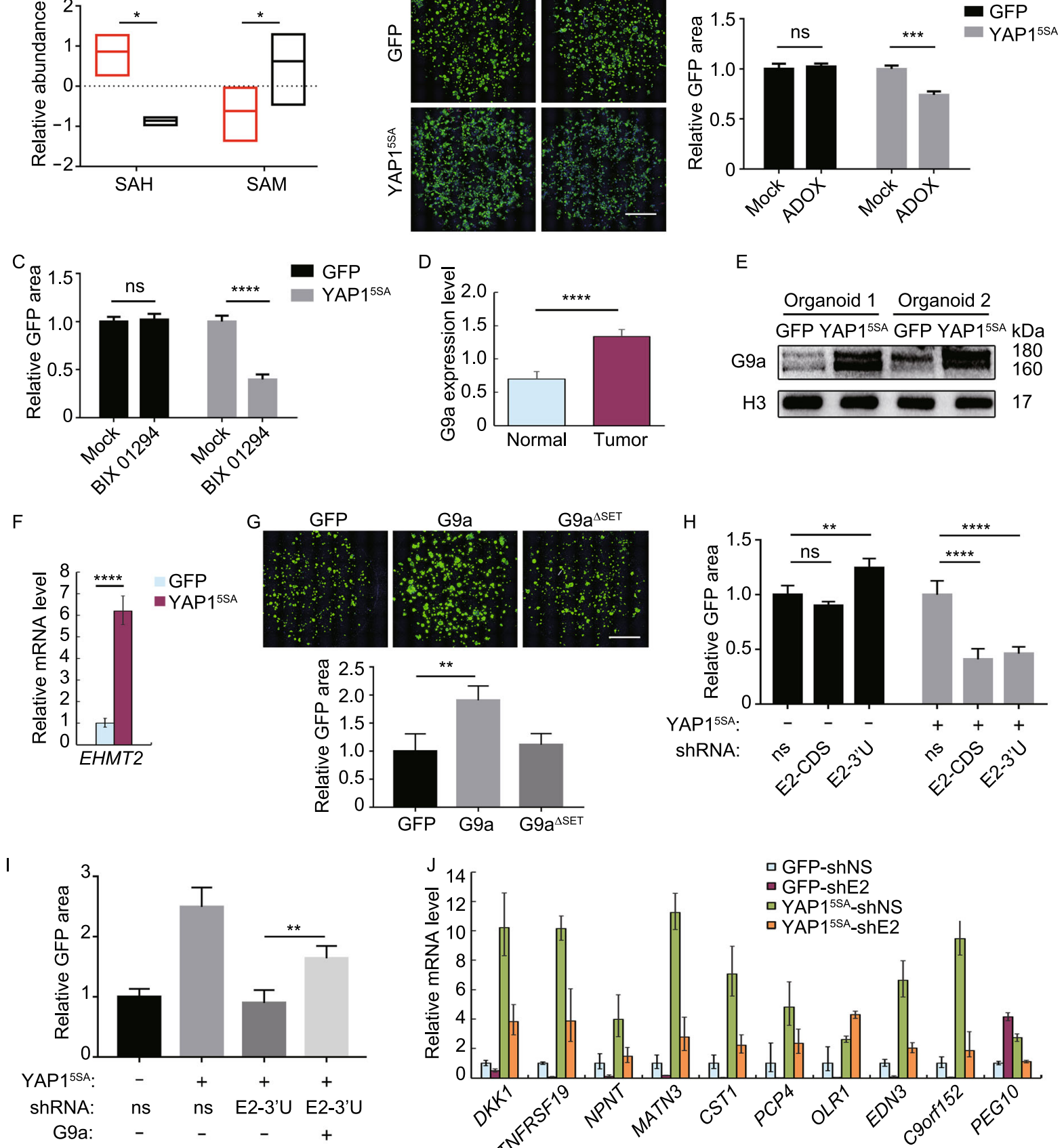

E

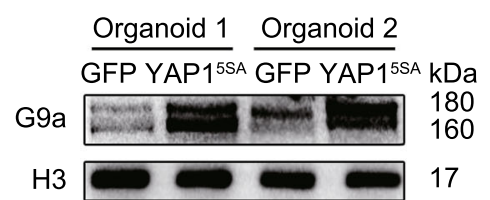

7
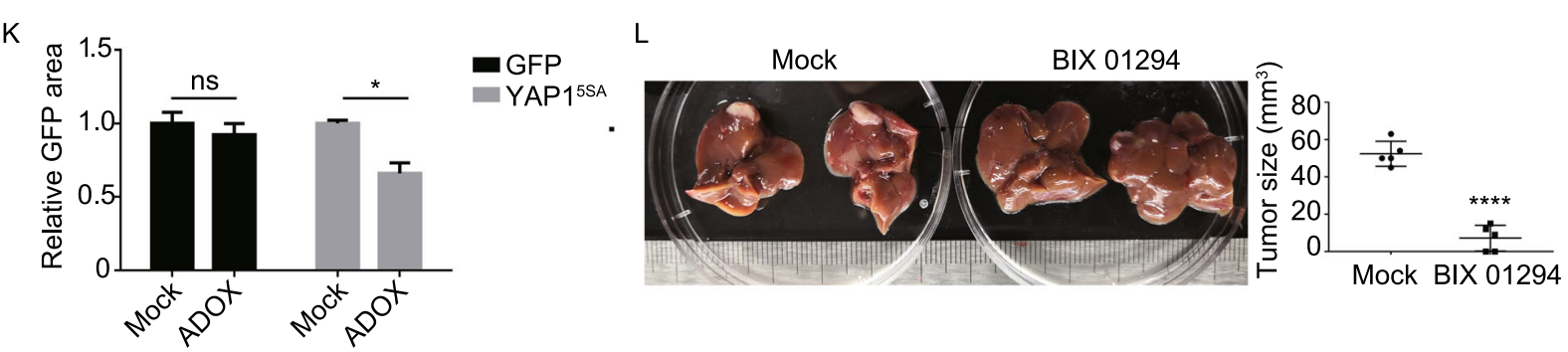
Figure 2. YAP1 activation initiates hepatoblastoma through up-regulating methyltransferase G9a. (A) Metabolomic analysis revealed that S-adenosylmethionine (SAM) was downregulated while S-adenosyl-L-homocysteine (SAH) was upregulated in YAP1-activated $\mathrm{HB}$ organoids. (B) ADOX treatment for 7 days specifically inhibited the growth of YAP1-activated HB organoids. Data were presented as means \pm SD $(n=4)$. Scale bar, $1 \mathrm{~mm}$. (C) BIX 01294 treatment for 7 days specifically inhibited the growth of YAP1-activated HB organoids. Data were presented as means $\pm \operatorname{SD}(n=4)$. (D) The data from HBprem DataBase showed that G9a was up-regulated in HB at protein level when compared to normal tissue. Data were presented as means $\pm \operatorname{SD}(n=5)$. (E) Western blot determined the expression of G9a in two lines of organoids transfected with GFP or YAP1 ${ }^{5 S A}$. (F) qRT-PCR analysis of EHMT2 expression in organoids transfected with GFP or YAP $1^{5 S A}$. $H 3$ was used as an internal control. Data were presented as means $\pm \operatorname{SD}(n=3)$. (G) Wild-type G9a but not its enzyme-dead mutant ( $\triangle \mathrm{SET}$ ) drove HB oncogenesis. Data were presented as means \pm SD $(n=4)$. Scale bar, $1 \mathrm{~mm}$. (H) Knockdown of EHMT2 by shRNAs inhibited the growth of YAP1-activated HB organoids. E2-CDS: EHMT2 shRNA targeting CDS; E2-3'U: EHMT2 shRNA targeting $3^{\prime} U T R$. Data were presented as means $\pm \operatorname{SD}(n=4)$. (I) Overexpression of G9a restored the growth of YAP1activated $\mathrm{HB}$ organoids transfected with EHMT2 shRNA targeting $3^{\prime} U T R$. Data were presented as means \pm SD $(n=$ 4). (J) qRT-PCR analysis revealed that EHMT2 knockdown down-regulated $H B$ signature genes in YAP1-activated HB organoids. $\mathrm{H} 3$ was used as an internal control. Data were presented as means $\pm \mathrm{SD}(n=3)$. (K) Human fetal liver organoids transfected with YAP $1^{5 S A}$ for 40 days to achieve HB tumorigenesis. These $\mathrm{HB}$ organoids were then treated with ADOX for 12 days. Data were presented as means $\pm \operatorname{SD}(n=4)$. (L) NSG mice were transplanted with YAP1-activated HB organoids to obtain liver tumors. Then, these mice were then received daily treatment of BIX $01294(5 \mathrm{mg} / \mathrm{kg})$ for 10 days. Data were presented as means $\pm \mathrm{SD}(n=5)$. ${ }^{*}$ Indicates $P<$ 0.05 ; ${ }^{* *}$ indicates $P<0.01$; ${ }^{* *}$ indicates $P<0.001 ;{ }^{* * * *}$ indicates $P<0.0001$.

Gynecology Hospital of Fudan University and with the Helsinki Declaration of 1975, as revised in 2000 (5). All fetuses were obtained with the informed consent by the patients who requested legally elective abortions at the Obstetrics and Gynecology Hospital of Fudan University.

All data and material are available from the corresponding authors upon reasonable request.

L.Y., J.L. and B.Z. conceived the study; L.Y., J.C., J.L., Y.Z., Q.W., X.R., J.W., Q.G., J.Z. and N.J. performed the experiments; X.L., J.L. and B.Z. supervised the work; L.Y., J.L. and B.Z. wrote the manuscript.
Li Yang ${ }^{1}$, Jin Chen ${ }^{1}$, Jianqing Liang ${ }^{1}$, Yufeng Zhang ${ }^{1}$, Qingzhe Wang ${ }^{2}$, Xiaojun Ren ${ }^{3}$, Jinsong Wei ${ }^{1}$, Qianchun Gong ${ }^{1}$, Jiting Zhang ${ }^{1}$, Ning Jiang ${ }^{1}$, Xinhua Lin ${ }^{1}$, Jin $\mathrm{Li}^{1 凶}$, Bing Zhao ${ }^{1 凶}$

${ }^{1}$ State Key Laboratory of Genetic Engineering, School of Life Sciences, Zhongshan Hospital, Fudan University, Shanghai 200438, China

2 bioGenous Biotechnology, Inc., Hangzhou 311231, China

${ }^{3}$ Obstetrics and Gynecology Hospital of Fudan University, Shanghai Key Laboratory of Female Reproductive Endocrine Related

Diseases, Fudan University, Shanghai 200011, China

$\bowtie$ Correspondence: Li_Jin_lifescience@fudan.edu.cn (J. Li), bingzhao@fudan.edu.cn (B. Zhao)

Accepted November 2, 2021

\section{OPEN ACCESS}

This article is licensed under a Creative Commons Attribution 4.0 International License, which permits use, sharing, adaptation, distribution and reproduction in any medium or format, as long as you give appropriate credit to the original author(s) and the source, provide a link to the Creative Commons licence, and indicate if changes were made. The images or other third party material in this article are included in the article's Creative Commons licence, unless indicated otherwise in a credit line to the material. If material is not included in the article's Creative Commons licence and your intended use is not permitted by statutory regulation or exceeds the permitted use, you will need to obtain permission directly from the copyright holder. To view a copy of this licence, visit http:// creativecommons.org/licenses/by/4.0/.

\section{REFERENCES}

Angelico R, Grimaldi C, Gazia C, Saffioti MC, Manzia TM, Castellano A, Spada M (2019) How do synchronous lung metastases influence the surgical management of children with hepatoblastoma? An update and systematic review of the literature. Cancers (basel) 11:1693

Driskill JH, Pan D (2021) The Hippo pathway in liver homeostasis and pathophysiology. Annu Rev Pathol 16:299-322

Hu H, Gehart H, Artegiani B, Lopez-lglesias C, Dekkers F, Basak O, van Es J, de Sousa C, Lopes SM, Begthel $\mathrm{H}$, Korving $\mathrm{J}$ et al (2018) Long-term expansion of functional mouse and human hepatocytes as 3D organoids. Cell 175:1591-1606

O'Neill AF, Towbin AJ, Krailo MD, Xia CH, Gao Y, McCarville MB, Meyers RL, McGahren ED, Tiao GM, Dunn SP et al (2017) Characterization of pulmonary metastases in children with hepatoblastoma treated on Children's Oncology Group protocol AHEP0731 (the treatment of children with all stages of hepatoblastoma): a report from the Children's Oncology Group. J Clin Oncol 35:3465 
Prior N, Hindley CJ, Rost F, Melendez E, Lau WWY, Gottgens B, Rulands S, Simons BD, Huch M (2019) Lgr5(+) stem and progenitor cells reside at the apex of a heterogeneous embryonic hepatoblast pool. Development 146:dev174557

Sumazin P, Chen Y, Trevino LR, Sarabia SF, Hampton OA, Patel K, Mistretta TA, Zorman B, Thompson P, Heczey A et al (2017) Genomic analysis of hepatoblastoma identifies distinct molecular and prognostic subgroups. Hepatology 65:104-121

Sun L, Wang Y, Cen J, Ma X, Cui L, Qiu Z, Zhang Z, Li H, Yang RZ, Wang $C$ et al (2019) Modelling liver cancer initiation with organoids derived from directly reprogrammed human hepatocytes. Nat Cell Biol 21:1015-1026

Sylvester KG, Colnot S (2014) Hippo/YAP, beta-catenin, and the cancer cell: a "menage a trois" in hepatoblastoma. Gastroenterology 147:562-565
Tao J, Calvisi DF, Ranganathan S, Cigliano A, Zhou L, Singh S, Jiang L, Fan B, Terracciano L, Armeanu-Ebinger S et al (2014) Activation of beta-catenin and Yap1 in human hepatoblastoma and induction of hepatocarcinogenesis in mice. Gastroenterology 147:690-701

Zhang Y, Solinas A, Cairo S, Evert M, Chen X, Calvisi DF (2021) Molecular mechanisms of hepatoblastoma. Semin Liver Dis 41:28-41

Zhao B, Wei X, Li W, Udan RS, Yang Q, Kim J, Xie J, Ikenoue T, Yu $\mathrm{J}$, Li L et al (2007) Inactivation of YAP oncoprotein by the Hippo pathway is involved in cell contact inhibition and tissue growth control. Genes Dev 21:2747-2761 